\title{
Correlation of Overexpression of Nestin with Expression of Epithelial-Mesenchymal Transition-Related Proteins in Gastric Adenocarcinoma
}

\author{
Jin-Kai Liu ${ }^{1 \&}$, Wan-Cheng Chen ${ }^{2 \&}$, Xiao-Zhen Ji ${ }^{1}$, Wen-Hong Zheng ${ }^{1}$, Wei Han ${ }^{1}$, \\ Jing An ${ }^{1 *}$
}

\begin{abstract}
Background: Nestin is associated with neoplastic transformation. However, the mechanisms by which nestin contributes regarding invasion and malignancy of gastric adenocarcinoma (GAC) remain unknown. Recent studies have shown that the epithelial-mesenchymal transition (EMT) is important in invasion and migration of cancer cells. In the present study, we aimed to investigate the expression of nestin and its correlation with EMT-related proteins in GAC. Materials and Methods: The expression of nestin and EMT-related proteins was examined in GAC specimens and cell lines by immunohistochemistry and Western blotting. Clinicopathological features and survival outcomes were retrospectively analyzed. Results: Positive nestin immunostaining was most obviously detected in the cytoplasm, nucleus or both cytoplasm and nucleus of tumor cells in $19.2 \%(24 / 125)$ of GAC tissues, which was significantly higher than that in normal gastric mucosa tissues $(1.7 \%, 1 / 60)(p=0.001)$. Nestin expression was closely related to several clinicopathological factors and EMT-related proteins (E-cadherin, vimentin and Snail) and displayed a poor prognosis. Interestingly, simultaneous cytoplasmic and nuclear nestin expression correlated with EMT-related proteins (E-cadherin, vimentin and Snail) $(p<0.05)$ and lymph node metastasis $(p=0.041)$ and a shorter survival time $(p<0.05)$, but this was not the case with cytoplasmic or nuclear nestin expression. Conclusions: Nestin, particularly expression in both cytoplasm and nucleus, might be involved in regulating EMT and malignant progression in GAC, with potential as an unfavorable indicator in tumor diagnosis and a target for clinical therapy.
\end{abstract}

Keywords: Nestin - epithelial-mesenchymal transition - gastric adenocarcinoma - immunohistochemistry - prognosis

Asian Pac J Cancer Prev, 16 (7), 2777-2783

\section{Introduction}

Gastric cancer is the fourth most common malignant disease and the second most frequent cause of cancerrelated death worldwide (Takaishi et al., 2009). More than $95 \%$ of gastric cancer cases are adenocarcinomas. Despite significant advancements in diagnosis and treatment modalities, especially surgery, chemotherapy, and radiotherapy, the outcome for patients with gastric cancer remains poor, with a 5-year survival rate of $20 \%$. The poor prognosis of gastric cancer is due to the high recurrence rates, nodal metastasis, and short-lived response to chemotherapy (Hartgrink et al., 2009; Ni et al., 2013).

The epithelial-mesenchymal transition (EMT) is a critical biological process by which epithelial cells lose their polarized, epithelial morphology and acquire a mesenchymal-like phenotype. It is characterized by the downregulation of epithelial protein expression, such as E-cadherin, and the upregulation of mesenchymal protein expression, such as vimentin and fibronectin (Luo et al.,
2012; Zhu et al., 2013). Loss of functional expression of E-cadherin protein and/or transcriptional repression of its mRNA is considered a hallmark of EMT (Kang and Massague, 2004). Vimentin is an intermediate filament (IF) protein that is a component of the cytoskeleton, and is normally expressed in mesenchymal cells but not usually in epithelial cells (Prudkin et al., 2009). Snail, a SNAG domain-containing zinc finger protein, directly binds to the E-boxes in the promoter of the E-cadherin gene to repress its transcription and convert normal epithelial cells into the mesenchymal cell phenotype (Batlle et al., 2000). Several distinct cellular properties are conferred during EMT, including cell motility and invasiveness.

Nestin, a cytoskeleton-associated class VI IF protein, is a neuronal stem/progenitor cell marker that is expressed in progenitor cells of various tissues, including brain tumors (Thomas et al., 2004), pancreas (Matsuda et al., 2012; Kim et al., 2012), malignant melanoma (Laga et al., 2011) skin tumor (Sabet et al., 2014) and prostate cancer (Gu et al., 2007). Recent reports support a link between nestin and malignant characteristics and suggest that abundant 


\section{Jin-Kai Liu et al}

nestin expression is correlated with greater malignancy and poorer prognosis in different cancers (Klein et al., 2007; Yang et al., 2008; Piras et al., 2011; Ishiwata et al., 2011). Knockdown of nestin in lung adenocarcinoma cells resulted in decreased proliferation, migration, invasion, and sphere formation, while upregulation by nestin gene transfection produced the opposite effects (Narita et al., 2014). Sen et al. suggested that patients with positive nestin expression in high grade bladder cancer tissues need close follow-up and might be informed about more tendency to recur (Sen et al., 2014). These findings above indicate that nestin is important in the aggressiveness of cancer cells. However, up to date, the specific function of nestin in the invasive and metastatic behaviors of gastric cancer cells remains poorly understood.

In the present study, we investigated the expression and prognostic impact of nestin in a gastric adenocarcinoma (GAC) cohort and examined the possible correlation between nestin and the EMT-related proteins E-cadherin, vimentin and Snail.

\section{Materials and Methods}

\section{Tissue samples}

A total of 125 GAC tissue samples were acquired from the Nanfang Hospital, Southern Medical University, Guangzhou, China between August 2006 to October 2007. No patients received preoperative radiotherapy or chemotherapy. Subjects comprised 97 males and 28 females, with ages ranging from 39 to 85 years (median, 62.1 years). Patient data were analyzed after 5 years of follow-up. The median follow-up time was 38.6 months (range, 3-74 months). The end date of follow-up was October 2012. Control normal gastric mucosa samples were collected from 60 cases during operation at a distance of $5 \mathrm{~cm}$ from the tumor. Prior patient consent was obtained and approval was confirmed from the ethics committee of South Medical University.

\section{Immunohistochemistry}

Paraffin-embedded samples were serially sectioned at $3 \mu \mathrm{m}$ thickness. Each section was dewaxed in xylene for $30 \mathrm{~min}$ and rehydrated in a graded series of alcohol. The slides were then placed in citrate buffer for $2 \mathrm{~min}$ at $100^{\circ} \mathrm{C}$ for antigen retrieval. After natural cooling, $3 \%$ hydrogen peroxide and serum were used to block endogenous peroxidase activity and non-specific antigen. Primary antibodies nestin (monoclonal mouse, dilution 1/400; Abcam, New Territories, HK), E-cadherin (monoclonal rabbit, dilution 1/500; Abcam, Cambridge, MA, USA), vimentin (monoclonal rabbit, dilution 1/400; Abcam, Cambridge, MA, USA), and Snail (monoclonal rabbit, dilution 1/100; Cell Signaling, Beverly, MA, USA) were incubated overnight at $4^{\circ} \mathrm{C}$. After washing in phosphatebuffered saline (PBS) buffer, the sections were incubated with peroxidase-conjugated anti-mouse/rabbit secondary antibodies at $37^{\circ} \mathrm{C}$ for $30 \mathrm{~min}$. The peroxidase reaction was developed using 3,3'-diaminobenzidine (DAB) for 1 min. Sections were then counterstained with hematoxylin, dehydrated and analyzed using a light microscope. In every run, the primary antibody was substituted with PBS as a negative control.

\section{Cell culture}

The human GAC cell lines MKN28 and AGS were obtained from the Cancer Research Institute, Southern Medical University (Guangzhou, China). The cells were cultured in PRMI 1640 (Hyclone, Waltham, MA, USA) supplemented with $10 \%$ fetal bovine serum (Hyclone) in a humidified atmosphere of $37^{\circ} \mathrm{C}$ at $5 \% \mathrm{CO}_{2}$.

\section{Transfection}

The siRNAs targeting human nestin (Gene ID: 10763) mRNA (si-nestin) and NCsiRNA (si-Control) were purchased from RiboBio (Guangzhou, China). MKN28 and AGS cells were seeded for $12 \mathrm{~h}$ and transfected using the C10511-05 riboFect ${ }^{\mathrm{TM}}$ CP Transfection Kit (166T) (RiboBio) according to the manufacturer's instructions.

\section{Western blotting}

Proteins were separated by SDS-PAGE and electrotransferred to membranes. Membranes were blocked with a solution of TBS/0.1\% Tween-20 and incubated with primary antibodies nestin (monoclonal mouse, dilution 1/200; Abcam, New Territories, HK), E-cadherin (monoclonal rabbit, dilution 1/1000; Abcam, Cambridge, MA, USA), vimentin (monoclonal rabbit, dilution 1/1000; Abcam, Cambridge, MA, USA), and Snail (monoclonal rabbit, dilution 1/600; Cell Signaling, Beverly, MA, USA) overnight, followed by incubation with the appropriate horseradish peroxidase conjugated anti-mouse/rabbit secondary antibody (Cell Signaling Tech, Beverly, MA, USA). $\beta$-Actin (monoclonal mouse, dilution 1/200; Santa Cruz, CA, USA) was used as an internal control. Protein visualization was performed by enhanced chemiluminescence (KeyGEN Bio).

\section{Evaluation of immunostaining}

Tissue sections stained immunohistochemically for nestin and EMT-related proteins were reviewed separately by two pathologists blinded to the clinical parameters. A semi-quantitative approach was used to score the immunoassay. For nestin staining, samples were graded according to the percentage of positive cells as follows: 0 points $=0-5 \%, 2$ points $=6-50 \%$, and 3 points $=$ more than $50 \%$. The staining intensity was rated as follows: 3 points for strong intensity, 2 points for moderate intensity, or 1 point for weak intensity. Adding the percentage and intensity scores provided a composite expression score (1 to 6); a sum of scores $\geq 3$ points was considered positive, whereas a sum of scores $<3$ points was regarded as negative (Kim et al., 2012; He et al., 2013).

E-cadherin staining is localized on the membrane or in the cytoplasm. In our study, all specimens without membranous E-cadherin expression were regarded as negative (Zhou et al., 2007). "Positive (intact)" E-cadherin in GAC tissues was defined when over $25 \%$ of the cancer cells showed immunoreactivity on the cell membrane (Ryu et al., 2012).

Vimentin is highly expressed in cancer stromal and inflammatory cells, but is not usually present in epithelial cells. Therefore, the expression of vimentin 
Overexpression of Nestin and Epithelial-Mesenchymal Transition-Related Proteins in Gastric Adenocarcinoma

was considered to be positive when $>10 \%$ of tumor cells showed moderate staining (Kim et al., 2009; Hou et al., 2012). This scoring criterion was also applied to Snail (Kim et al., 2009; Luo et al., 2012).

\section{Statistical analysis}

All statistical analyses were analyzed using SPSS13.0 software (SPSS Inc., Chicago, IL, USA). The chisquare test and Fisher's exact test (when cells have an expected count of less than five) were used to analyze the relationships between levels of nestin expression and clinicopathological characteristics. The overall survival rate was estimated using the Kaplan-Meier method and compared using the log-rank test. The Cox proportional hazards regression model was applied for the multivariate analysis. Correlations between variable factors were calculated by Spearman correlation coefficients. Statistical significance was considered at $p<0.05$.

\section{Results}

Expression and localization of nestin, E-cadherin, vimentin and Snail in gastric adenocarcinoma and normal gastric mucosa

The expression levels of nestin protein were examined in 125 paraffin-embedded human GAC samples and 60 normal gastric mucosa samples by immunohistochemical staining. Positive nestin immunostaining was observed in $19.2 \%(24 / 125)$ GAC cases, which was significantly higher than that in normal gastric mucosa samples $(1.7 \%$; $1 / 60)(p=0.001)$ (Table 1). Nestin staining in GAC cases showed cytoplasmic localization, nuclear localization or staining in both cytoplasm and nuclei (Figure 1A-D).

Positive membranous expression of E-cadherin was detected in $53.6 \%(67 / 125)$ of GAC cases and $90.0 \%$ $(54 / 60)$ of normal gastric mucosa samples. Through the $\chi^{2}$-test, a significant difference was displayed in these two groups $(p=0.000)$ (Table 1). Representative immunohistochemical staining of E-cadherin is shown in Figure 1E and F.
Strong cytoplasmic localization of vimentin was detected in $12.8 \%(16 / 125)$ of tumor cases. In contrast, no cytoplasmic vimentin expression was observed in normal gastric mucosa, and vimentin was mainly expressed in stromal and inflammatory cells. The difference in vimentin positive expression between the cancer group and the normal gastric mucosa group was significant $(p=0.004)$ (Table 1). Vimentin immunohistochemical staining is shown in Figure $1 \mathrm{G}$ and $\mathrm{H}$.

Snail positive expression, found in both the cytoplasm and nucleus of tumor cells, was observed in $20.8 \%$ (26/125) of gastric cancer tissues. Some of the tumors had large solid areas of malignant cells that showed no increase in Snail expression within the central region of the cells but a tendency for increased expression in the peripheral layers of cells. Malignant cells at the invasive edge of the tumor tended to show increased staining that was more pronounced if the invasive edge was penetrating the subserosal tissue. However, in normal gastric mucosa tissues, only $3.3 \%$ (2/60) cases displayed Snail positive expression. The $\chi^{2}$-test indicated a significant difference in Snail positive expression between the cancer group

Table 1. Expression of Nestin and EMT-Related Proteins in Gastric Adenocarcinoma and Normal Gastric Mucosa Tissues

\begin{tabular}{lcccc}
\hline Groups & $\begin{array}{c}\text { Cancer } \\
\text { tissues(\%) }\end{array}$ & $\begin{array}{c}\text { Normal } \\
\text { mucosa (\%) }\end{array}$ & $\chi^{2}$ & $P$ \\
\hline Nestin & & & & \\
Positive & $24(19.2)$ & $1(1.7)$ & 10.664 & 0.001 \\
$\begin{array}{c}\text { Negative } \\
\text { E-cadherin }\end{array}$ & $101(80.8)$ & $59(98.3)$ & & \\
Positive & $67(53.6)$ & $54(90.0)$ & 23.739 & 0.000 \\
Negative & $58(46.4)$ & $6(10.0)$ & & \\
Vimentin & & & & \\
Positive & $16(12.8)$ & $0(0)$ & 8.407 & 0.004 \\
Negative & $109(87.2)$ & $60(98.3)$ & & \\
Snail & & & & \\
Positive & $26(20.8)$ & $2(3.3)$ & 9.629 & 0.002 \\
Negative & $99(79.2)$ & $58(96.7)$ & & \\
\hline
\end{tabular}

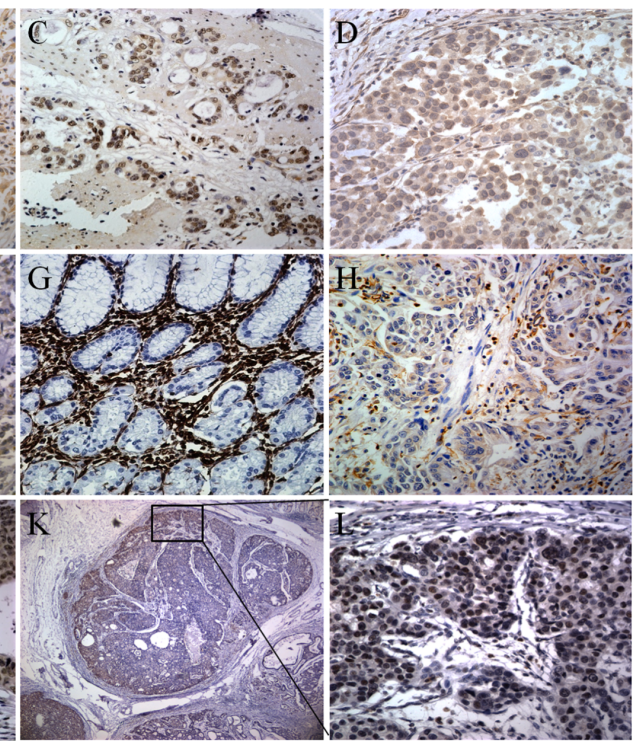

Figure 1. Immunostaining of Nestin, E-cadherin, Vimentin and Snail in Gastric Adenocarcinoma and Normal Gastric Mucosa Tissues 
Jin-Kai Liu et al

and the normal gastric mucosa group $(p=0.002)$ (Table 1). The immunohistochemical staining of Snail is shown in Figure 1I-L.

Associations between nestin with clinicopathological features and EMT-related proteins in gastric adenocarcinoma

As summarized in Table 2, the overexpression of nestin was closely related to the tumor size $(p=0.023)$, $\mathrm{T}$ classification $(p=0.003), \mathrm{N}$ classification $(p=0.033)$ and pTNM stage $(p=0.023)$. However, no significant correlations were observed with patient age, gender and histologic grade $(p>0.05)$. Moreover, the positive expression of nestin was negatively correlated with E-cadherin expression $(\mathrm{r}=-0.280, p=0.002)$ and positively associated with vimentin $(\mathrm{r}=0.482, p=0.000)$ and Snail $(\mathrm{r}=0.451, p=0.000)$ expression.

Next, we analyzed the correlation between cytoplasmic nestin, nuclear nestin and both cytoplasmic and nuclear nestin expression with the clinicopathological factors and EMT markers of GAC. As shown in Table 3, patients with cytoplasmic nestin expression showed a positive correlation with vimentin expression $(\mathrm{r}=0.427, p=0.000)$ and Snail expression $(\mathrm{r}=0.304, p=0.004)$, but there was no significant correlation with E-cadherin expression $(p=0.703)$. The group with nuclear nestin expression

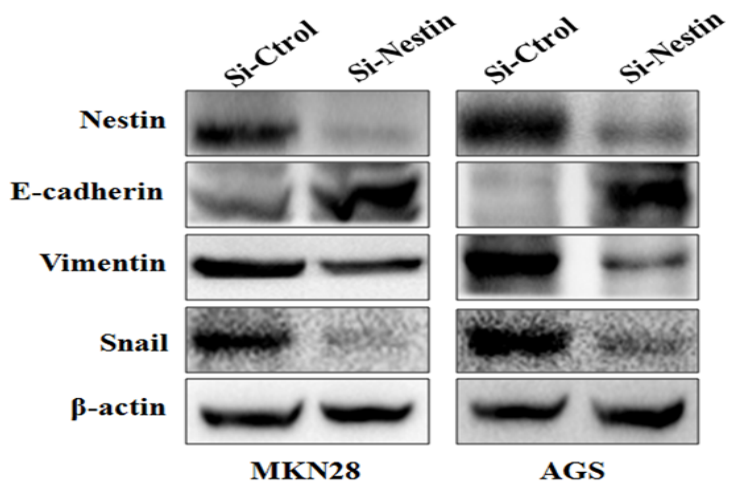

Figure 2. SiRNA-Mediated Silencing of Nestin Influenced Expression of EMT-Related Proteins

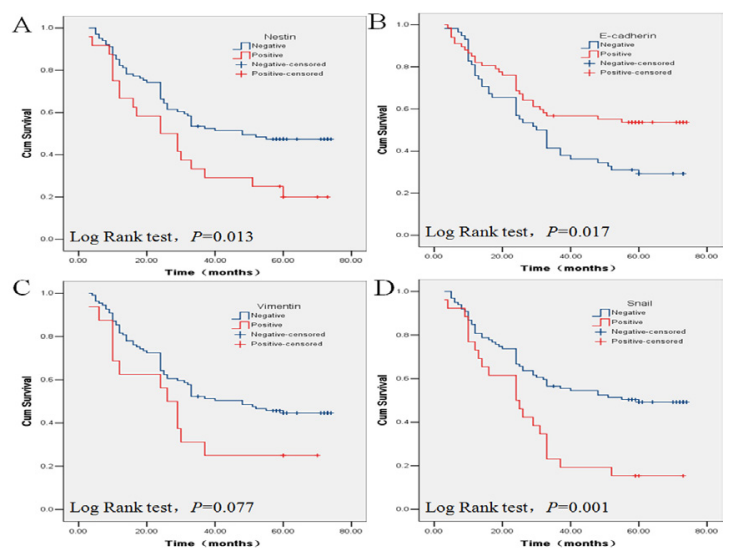

Figure 3. Overall survival curves of patients with gastric adenocarcinoma according to the immunostaining results of nestin, E-cadherin, vimentin and Snail determined by Kaplan-Meier analysis
Table 2. Correlations between Nestin Expression with Clinicopathologic Characteristics and Emt-Related Proteins

\begin{tabular}{|c|c|c|c|c|c|}
\hline \multirow[t]{2}{*}{ Variables } & \multirow[t]{2}{*}{$n$} & \multicolumn{4}{|c|}{ Nestin } \\
\hline & & Positive & Negative & $\chi^{2}$ & $P$ \\
\hline \multicolumn{6}{|l|}{ Gender } \\
\hline Male & 97 & 16 & 81 & 2.043 & 0.153 \\
\hline Female & 28 & 8 & 20 & & \\
\hline \multicolumn{6}{|l|}{ Age(years) } \\
\hline$<60$ & 55 & 10 & 45 & 0.066 & 0.798 \\
\hline$\geq 60$ & 70 & 14 & 56 & & \\
\hline \multicolumn{6}{|l|}{ Tumor size $(\mathrm{cm})$} \\
\hline$<5$ & 40 & 3 & 37 & 5.191 & 0.023 \\
\hline$\geq 5$ & 85 & 21 & 64 & & \\
\hline \multicolumn{6}{|l|}{$\mathrm{T}$ classification } \\
\hline $\mathrm{T} 1-\mathrm{T} 2$ & 29 & 0 & 29 & 8.973 & 0.003 \\
\hline $\mathrm{T} 3-\mathrm{T} 4$ & 96 & 24 & 72 & & \\
\hline \multicolumn{6}{|l|}{$\mathrm{N}$ classification } \\
\hline N0-N1 & 50 & 5 & 45 & 4.547 & 0.033 \\
\hline $\mathrm{N} 2-\mathrm{N} 3$ & 75 & 19 & 56 & & \\
\hline \multicolumn{6}{|l|}{ pTNM stage } \\
\hline I-II & 40 & 3 & 37 & 5.191 & 0.023 \\
\hline III-IV & 85 & 21 & 64 & & \\
\hline \multicolumn{6}{|l|}{ Histology } \\
\hline Well-Moderate & 63 & 8 & 55 & 3.461 & 0.063 \\
\hline Poor-Undifferentiated & 62 & 16 & 46 & & \\
\hline \multicolumn{6}{|c|}{ E-cadherin } \\
\hline Positive & 67 & 6 & 61 & 9.769 & 0.002 \\
\hline Negative & 58 & 18 & 40 & & \\
\hline \multicolumn{6}{|l|}{ Vimentin } \\
\hline Positive & 16 & 11 & 5 & 29.039 & 0.000 \\
\hline Negative & 109 & 13 & 96 & & \\
\hline \multicolumn{6}{|l|}{ Snail } \\
\hline Positive & 26 & 14 & 12 & 25.401 & 0.000 \\
\hline Negative & 99 & 10 & 89 & & \\
\hline
\end{tabular}

showed association with E-cadherin downregulation $(\mathrm{r}=-0.199, p=0.044)$ and Snail upregulation $(\mathrm{r}=0.218$, $p=0.034$ ), whereas no statistical difference was observed with the expression of vimentin $(p=1.000)$. Notably, patients with both cytoplasmic and nuclear nestin expression were negatively correlated with E-cadherin expression $(\mathrm{r}=-0.192, p=0.049)$ and positively associated with vimentin ( $\mathrm{r}=0.427, p=0.000)$ and Snail expression $(\mathrm{r}=0.212, p=0.032)$ and lymph node metastasis $(p=0.041)$. Furthermore, patients with both cytoplasmic and nuclear nestin expression displayed a shorter survival time $(p=0.041)$.

SiRNA-mediated silencing of nestin influenced expression of EMT-related proteins in human gastric adenocarcinoma cells in vitro

To further examine the relationship between nestin and EMT-related proteins, nestin expression was knocked down with lentivirus-mediated shRNA in MKN28 and AGS cells in vitro (Figure 2). Compared with cells infected with the control shRNA lentivirus, cells infected with nestin shRNA lentivirus exhibited increased E-cadherin expression but reduced vimentin and Snail expression.

Survival analysis according to nestin and EMT-related proteins expression

Kaplan-Meier analysis and the log-rank test were 
Table 3. Correlation between the Clinicopathologic Characteristics and Expression of Nestin in Cytoplasm, Nucleus and in both Cytoplasm and Nucleus of 125 Gastric Adenocarcinoma Patients

\begin{tabular}{|c|c|c|c|c|c|c|c|c|c|c|}
\hline \multirow{3}{*}{ Variables } & \multirow{3}{*}{$n$} & \multicolumn{9}{|c|}{ Nestin expression } \\
\hline & & \multicolumn{3}{|c|}{ Cytoplasm } & \multicolumn{3}{|c|}{ Nucleus } & \multicolumn{3}{|c|}{ Cytoplasm\&Nucleus } \\
\hline & & + & - & $P$ & + & - & $P$ & + & - & $P$ \\
\hline \multicolumn{11}{|l|}{ Gender } \\
\hline Male & 97 & 4 & 93 & \multirow[t]{2}{*}{0.186} & 7 & 90 & \multirow[t]{2}{*}{0.692} & 5 & 92 & \multirow[t]{2}{*}{0.653} \\
\hline Female & 28 & 3 & 25 & & 3 & 25 & & 2 & 26 & \\
\hline \multicolumn{11}{|l|}{ Age } \\
\hline$<60$ & 55 & 2 & 53 & \multirow[t]{2}{*}{0.464} & 5 & 50 & \multirow[t]{2}{*}{0.748} & 3 & 52 & \multirow[t]{2}{*}{1.000} \\
\hline$\geq 60$ & 70 & 5 & 65 & & 5 & 65 & & 4 & 66 & \\
\hline \multicolumn{11}{|l|}{ Tumor size $(\mathrm{cm})$} \\
\hline$<5$ & 40 & 2 & 38 & \multirow[t]{2}{*}{1.000} & 1 & 39 & \multirow[t]{2}{*}{0.166} & 0 & 40 & \multirow[t]{2}{*}{0.096} \\
\hline$\geq 5$ & 85 & 5 & 80 & & 9 & 76 & & 7 & 78 & \\
\hline \multicolumn{11}{|l|}{$\mathrm{T}$ classification } \\
\hline $\mathrm{T} 1-\mathrm{T} 2$ & 29 & 0 & 29 & \multirow[t]{2}{*}{0.199} & 0 & 29 & \multirow[t]{2}{*}{0.115} & 0 & 29 & \multirow[t]{2}{*}{0.199} \\
\hline T3-T4 & 96 & 7 & 89 & & 10 & 86 & & 7 & 89 & \\
\hline \multicolumn{11}{|l|}{$\mathrm{N}$ classification } \\
\hline N0-N1 & 50 & 2 & 48 & \multirow[t]{2}{*}{0.701} & 3 & 47 & \multirow[t]{2}{*}{0.739} & 0 & 50 & \multirow[t]{2}{*}{0.041} \\
\hline N2-N3 & 75 & 5 & 70 & & 7 & 68 & & 7 & 68 & \\
\hline pTNM stage & & & & & & & & & & \\
\hline I-II & 40 & 0 & 40 & 0.096 & 3 & 37 & 1.000 & 0 & 40 & 0.096 \\
\hline III-IV & 85 & 7 & 78 & & 7 & 78 & & 7 & 78 & \\
\hline Histology & & & & & & & & & & \\
\hline Well-Moderate & 63 & 4 & 59 & 1.000 & 2 & 61 & 0.054 & 2 & 61 & 0.273 \\
\hline Poor-Undifferentiated & 62 & 3 & 59 & & 8 & 54 & & 5 & 57 & \\
\hline Survival time(years) & & & & & & & & & & \\
\hline$<5$ & 73 & 5 & 68 & 0.698 & 7 & 66 & 0.520 & 7 & 66 & 0.041 \\
\hline$\geq 5$ & 52 & 2 & 50 & & 3 & 49 & & 0 & 52 & \\
\hline E-cadherin & & & & & & & & & & \\
\hline Positve & 67 & 3 & 64 & 0.703 & 2 & 65 & 0.044 & 1 & 66 & 0.049 \\
\hline Negative & 58 & 4 & 54 & & 8 & 50 & & 6 & 52 & \\
\hline Vimentin & & & & & & & & & & \\
\hline Positve & 16 & 5 & 11 & 0.000 & 1 & 15 & 1.000 & 5 & 11 & 0.000 \\
\hline Negative & 109 & 2 & 107 & & 9 & 100 & & 2 & 107 & \\
\hline Snail & & & & & & & & & & \\
\hline Positive & 26 & 5 & 21 & 0.004 & 4 & 22 & 0.034 & 5 & 21 & 0.032 \\
\hline Negative & 99 & 2 & 97 & & 3 & 96 & & 5 & 94 & \\
\hline
\end{tabular}

used to detect the prognostic impact of nestin and EMT markers expression on patient survival. Patients with positive nestin and Snail staining had a more unfavorable prognosis than those with negative nestin and Snail expression ( $p=0.013$, Figure $3 \mathrm{~A} ; p=0.001$, Figure $3 \mathrm{D})$. Similarly, patients with a loss of E-cadherin expression also showed a shorter survival duration $(p=0.017$, Figure 3B). Positive vimentin expression had no significant impact on outcome, but a trend toward reduced survival was observed in patients with positive vimentin expression $(p=0.077$, Figure 3C).

Cox models were performed to determine the independent factors for GAC patients. Results showed that only $\mathrm{N}$ classification might be an independent prognostic factor for human GAC after resection $(R R=3.559$, $p=0.003$ ).

\section{Discussion}

Many recent studies demonstrated that high expression of nestin was associated with metastatic progression in pancreas (Cheng et al., 2013), prostate cancer (Kleeberger et al., 2007), glioma (Ishiwata et al., 2011), and lung cancer (Narita et al., 2014). However, it has not been clear whether nestin expression is clinically correlated with gastric cancer progression. To address this issue, we examined the expression of nestin in 125 human primary GAC samples using immunohistochemical analyses.

Our data suggested that the expression of nestin was significantly higher in GAC cases than in normal gastric mucosa samples, indicating that nestin might be involved in the pathogenesis of GAC. Furthermore, our results also revealed that the expression of nestin correlated with tumor size, $\mathrm{T}$ classification, $\mathrm{N}$ classification, and pTNM stage in GAC. Moreover, the overexpression of nestin was also found to be significantly associated with the prognosis of the patients who had undergone operation. These findings are consistent with other studies of breast cancer (Piras et al., 2011), non-small cell lung cancer (Chen et al., 2014), and melanomas (Florenes et al., 1994).

Although many reports have examined nestin expression in carcinomas, few studies have investigated the relationship between nestin and EMT in gastric cancer. As a feature of aggressive tumors, EMT is characterized by a switch from a polarized epithelial phenotype to mesenchymal properties. This endows tumor cells with 


\section{Jin-Kai Liu et al}

the capacity to invade surrounding tissues and metastasize (Guarino et al., 2007). Previous reports showed that Snail acts as a central transcription factor and can directly repress E-cadherin expression and play a key role in EMT (Savagner, 2001; Huber et al., 2005; Schaeffer et al., 2010). Hagio also suggested that Snail gene expression in pancreatic ductal adenocarcinoma cells was altered concomitantly with changes in nestin expression (Hagio et al., 2013). In our study, we evaluated three EMT markers (E-cadherin, vimentin and Snail) and found that nestin overexpression was associated with downregulation of E-cadherin and overexpression of the mesenchymal marker vimentin and the EMT transcription factor Snail. Western blotting confirmed these results, providing a potential mechanism by which nestin might promote the invasion and migration of GAC cells.

To the best of our knowledge, nestin is most commonly localized in the cytoplasm. However, in this study, we observed that nestin was localized in the cytoplasm and/ or nucleus of primary cancer cells, as described for other tumor cells (Veselska et al., 2006; Loja et al., 2009; Krupkova et al., 2011). Yang et al. reported that nestin was localized in the cytoplasm of most tumor cells, but in poorly differentiated angiosarcoma, some tumor cells expressed nestin in the nucleus as well (Yang et al., 2008). Krupkova et al. speculated that nuclear localization of nestin could be due to a dysfunction in certain signaling pathways or defective nestin structure in tumor cells (Krupkova et al., 2010). These findings, as well as results obtained by Thomas et al. and Hartig et al. support the hypothesis that nestin may have a function in chromatin remodeling and gene regulation (Hartig et al., 1998; Thomas et al., 2004). However, little is known about the effect of the differential localization of nestin and the classical nuclear localization signals in human tumors. Our data showed that nuclear nestin and both cytoplasmic and nuclear nestin expression were negatively correlated with E-cadherin which plays a major role in EMT. In contrast, no significant correlation was observed between cytoplasmic nestin expression and E-cadherin expression. More interestingly, patients with both cytoplasmic and nuclear nestin expression also correlated positively with mesenchymal marker vimentin expression and Snail expression, and showed a shorter survival time. Therefore, we hypothesized that nuclear localization of nestin might be an unfavorable indicator in tumor diagnosis. We also speculate that nestin, particularly localization of nestin in both cytoplasm and nucleus, might be involved in the regulation of EMT and malignant progression in GAC. More functional investigations are required to examine this hypothesis, and the mechanism of interaction between nuclear localization of nestin and E-cadherin should be investigated in future studies.

The univariate analysis suggested that GAC patients with positive nestin expression had shorter survival rates than those with negative nestin expression. However, multivariate Cox analysis demonstrated that nestin might not be an independent prognostic factor in GAC. Additionally, negative E-cadherin expression and positive Snail expression also correlated with a poor outcome for GAC patients, which is consistent with previous reports by Wang Wang et al. (2014).

In summary, the results of our study illuminated the association of a high expression level of nestin with E-cadherin downregulation, and vimentin and Snail expression in GAC. Nestin, especially cytoplasmic and nuclear nestin localization, might play a role in regulating the EMT and malignant progression in GAC. These findings may provide a molecular basis for restoring sensitivity to clinical therapies.

\section{Acknowledgements}

This work was supported by funding from the National 863 High Technology Research and Development program of China (no. 2006AA02A404).

\section{References}

Batlle E, Sancho E, Franci C, et al (2000). The transcription factor snail is a repressor of E-cadherin gene expression in epithelial tumour cells. Nat Cell Biol, 2, 84-9.

Chen Z, Wang J, Cai L, et al (2014). Role of the stem cellassociated intermediate filament nestin in malignant proliferation of non-small cell lung cancer. PLoS One, 9 , 85584 .

Cheng KH, Su HT, Weng CC, et al (2013). Stem Cell Marker Nestin is Critical for TGF Beta1-Mediated Tumor Progression in Pancreatic Cancer. Mol Cancer Res.

Florenes VA, Holm R, Myklebost O, Lendahl U, Fodstad O (1994). Expression of the neuroectodermal intermediate filament nestin in human melanomas. Cancer Res, 54, 354-6.

Gu G, Yuan J, Wills M, Kasper S (2007). Prostate cancer cells with stem cell characteristics reconstitute the original human tumor in vivo. Cancer Res, 67, 4807-15.

Guarino M, Rubino B, Ballabio G (2007). The role of epithelialmesenchymal transition in cancer pathology. Pathol, 39, 305-18.

Hagio M, Matsuda Y, Suzuki T, Ishiwata T (2013). Nestin regulates epithelial-mesenchymal transition marker expression in pancreatic ductal adenocarcinoma cell lines. Mol Clin Oncol, 1, 83-7.

Hartgrink HH, Jansen EP, van Grieken NC, van de Velde CJ (2009). Gastric cancer. Lancet, 374, 477-90.

Hartig R, Shoeman RL, Janetzko A, Tolstonog G, Traub P (1998). DNA-mediated transport of the intermediate filament protein vimentin into the nucleus of cultured cells. J Cell Sci, 111, 3573-84.

He QZ, Luo XZ, Zhou Q, et al (2013). Expression of nestin in ovarian serous cancer and its clinicopathologic significance. Eur Rev Med Pharmacol Sci, 17, 2896-901.

Hou F, Yuan W, Huang J, et al (2012). Overexpression of EphA2 correlates with epithelial-mesenchymal transition-related proteins in gastric cancer and their prognostic importance for postoperative patients. Med Oncol, 29, 2691-700.

Huber MA, Kraut N, Beug H (2005). Molecular requirements for epithelial-mesenchymal transition during tumor progression. Curr Opin Cell Biol, 17, 548-58.

Ishiwata T, Teduka K, Yamamoto T, et al (2011). Neuroepithelial stem cell marker nestin regulates the migration, invasion and growth of human gliomas. Oncol Rep, 26, 91-9.

Kang Y, Massague J (2004). Epithelial-mesenchymal transitions: twist in development and metastasis. Cell, 118, 277-9.

Kim HS, Yoo SY, Kim KT, et al (2012). Expression of the stem cell markers CD133 and nestin in pancreatic ductal adenocarcinoma and clinical relevance. Int J Clin Exp 
Pathol, 5, 754-61.

Kim MA, Lee HS, Lee HE, et al (2009). Prognostic importance of epithelial-mesenchymal transition-related protein expression in gastric carcinoma. Histopathol, 54, 442-51.

Kleeberger W, Bova GS, Nielsen ME, et al (2007). Roles for the stem cell associated intermediate filament Nestin in prostate cancer migration and metastasis. Cancer Res, 67, 9199-206.

Klein WM, Wu BP, Zhao S, et al (2007). Increased expression of stem cell markers in malignant melanoma. Mod Pathol, 20, 102-7.

Krupkova OJ, Loja T, Zambo I, Veselska R (2010). Nestin expression in human tumors and tumor cell lines. Neoplasma, 57, 291-8.

Krupkova OJ,Loja T, Redova M, et al (2011). Analysis of nuclear nestin localization in cell lines derived from neurogenic tumors. Tumour Biol, 32, 631-9.

Laga AC, Zhan Q, Weishaupt C, et al (2011). SOX2 and nestin expression in human melanoma: an immunohistochemical and experimental study. Exp Dermatol, 20, 339-45.

Loja T, Chlapek P, Kuglik P, et al (2009). Characterization of a GM7 glioblastoma cell line showing CD133 positivity and both cytoplasmic and nuclear localization of nestin. Oncol Rep, 21, 119-27.

Luo WR, Chen XY, Li SY, Wu AB, Yao KT (2012). Neoplastic spindle cells in nasopharyngeal carcinoma show features of epithelial-mesenchymal transition. Histopathol, 61, 113-22.

Matsuda Y, Kure S, Ishiwata T (2012). Nestin and other putative cancer stem cell markers in pancreatic cancer. Med Mol Morphol, 45, 59-65.

Narita K, Matsuda Y, Seike M, et al (2014). Nestin regulates proliferation, migration, invasion and stemness of lung adenocarcinoma. Int J Oncol, 44, 1118-30.

Ni C, Zhang Z, Zhu X, et al (2013). Prognostic value of CD166 expression in cancers of the digestive system: a systematic review and meta-analysis. PLoS One, 8, 70958.

Piras F, Ionta MT, Lai S, et al (2011). Nestin expression associates with poor prognosis and triple negative phenotype in locally advanced (T4) breast cancer. Eur J Histochem, 55, 39.

Prudkin L, Liu DD, Ozburn NC, et al (2009). Epithelial-tomesenchymal transition in the development and progression of adenocarcinoma and squamous cell carcinoma of the lung. Mod Pathol, 22, 668-78.

Ryu HS, Park DJ, Kim HH, Kim WH, Lee HS (2012). Combination of epithelial-mesenchymal transition and cancer stem cell-like phenotypes has independent prognostic value in gastric cancer. Hum Pathol, 43, 520-8.

Sabet MN, Rakhshan A, Erfani E, Madjd Z (2014). Coexpression of putative cancer stem cell markers, CD133 and Nestin, in skin tumors. Asian Pac J Cancer Prev, 15, 8161-9.

Savagner P (2001). Leaving the neighborhood: molecular mechanisms involved during epithelial-mesenchymal transition. Bioessays, 23, 912-23.

Schaeffer DF, Assi K, Chan K, et al (2010). Tumor expression of integrin-linked kinase (ILK) correlates with the expression of the E-cadherin repressor snail: an immunohistochemical study in ductal pancreatic adenocarcinoma. Virchows Arch, 456, 261-8.

Sen V, Bozkurt O, Demir O, et al (2014). Prognostic significance of nestin expression in pT1 high- grade bladder urothelial carcinoma patients treated with intravesical BCG. Asian Pac J Cancer Prev, 15, 10813-7.

Takaishi S, Okumura T, Tu S, et al (2009). Identification of gastric cancer stem cells using the cell surface marker CD44. Stem Cells, 27, 1006-20.

Thomas SK, Messam CA, Spengler BA, Biedler JL, Ross RA (2004). Nestin is a potential mediator of malignancy in human neuroblastoma cells. J Biol Chem, 279, 27994-9.
Veselska R, Kuglik P, Cejpek P, et al (2006). Nestin expression in the cell lines derived from glioblastoma multiforme. $B M C$ Cancer, 6, 32.

Wang ZS, Shen Y, Li X, et al (2014). Significance and prognostic value of Gli-1 and Snail/E-cadherin expression in progressive gastric cancer. Tumour Biol, 35, 1357-63.

Yang XH, Wu QL, Yu XB, et al (2008). Nestin expression in different tumours and its relevance to malignant grade. $J$ Clin Pathol, 61, 467-73.

Zhou Y, Ran J, Tang C, et al (2007). Effect of celecoxib on E-cadherin, VEGF, Microvessel density and apoptosis in gastric cancer. Cancer Biol Ther, 6, 269-75.

Zhu QC, Gao RY, Wu W, Qin HL (2013). Epithelialmesenchymal transition and its role in the pathogenesis of colorectal cancer. Asian Pac J Cancer Prev, 14, 2689-98. 